\title{
Dependence of the collision integral on electric field
}

\author{
I.I. Boiko \\ V. Lashkaryov Institute of Semiconductor Physics, NAS of Ukraine, \\ 45, prospect Nauky, 03028 Kyiv, Ukraine, \\ E-mail: igor.boiko.35@mail.ru \\ Phone: (380-44) 236-5422
}

\begin{abstract}
Conductivity of charged band carriers is considered for the case when collision integral evidently depends on electric field. This dependence for the case of scattering by charged impurities results in decrease of carrier conductivity.
\end{abstract}

Keywords: kinetic equation, collision integral, conductivity.

Manuscript received 13.11.14; revised version received 23.03.15; accepted for publication 27.05.15; published online 08.06.15.

\section{Introduction}

The collision integral (CI) is the principal term of kinetic equation. Its structure determines the whole form of the non-equilibrium function $f$ of many-body system. Usually, this integral is constructed for the second order of the perturbation theory, considering the interaction of charged carriers with stochastic microfields. On the way of consecutive deduction of CI from the "first principles" the specific term $E \cdot \delta \rho$ appears as an appendix to the particle energy; here, $E$ is the amplitude of electric field and $\delta \rho$ - fluctuation of the density matrix that is proportional to the scattering micropotential $\delta \varphi$. Most often, this term is omitted as the small one, together with terms of conformable higher orders for fluctuations. But any severe evidence of this neglect has not been represented and this trouble has not been overcome completely. The noted here problem is connected naturally with the specific question arising in quantum mechanics for the state of electron interacting with the constant uniform force $F$ (there was not represented the continuous analytical transition from the state at $F \neq 0$ to the state at $F=0)$. As a result, the influence of the maintained $(E \cdot \delta \varphi)$ term on the value of kinetic coefficients has not been well investigated up to this time.

\section{Fluctuative scattering potential and fluctuation of density matrix}

Introduce the one-particle density matrix of manyparticle system:

$\rho_{A B}(t)=a_{B}^{+}(t) a_{A}(t)$,

where $a_{A}^{+}$and $a_{A}$ are operators of generation and annihilation of a particle at the $A$ state. Heisenberg equation of motion for the density matrix is

$i \hbar \frac{\partial \rho_{A B}}{\partial t}=\left[\rho_{A B}(t), H^{t o t}\right]=\rho_{A B}(t) H^{t o t}-H^{t o t} \rho_{A B}(t)$

For the simplicity, restrict here our consideration by the case of electrons moving in uniform space at the presence of constant electric field $\vec{E}$ and set of charged impurities homogeneously distributed over the crystal. Here, total Hamiltonian is

$$
\begin{aligned}
& H^{\text {tot }}=\sum_{A} \varepsilon_{A} \rho_{A A}+e \sum_{A B} \varphi_{A B}^{(E)} \rho_{B A}+e \sum_{A B} \Phi_{A B}^{(S)} \rho_{B A}- \\
& (1 / 2) \sum_{A B A^{\prime} B^{\prime}} V_{A B A^{\prime} B^{\prime}}\left[\rho_{B^{\prime} A}, \rho_{B A^{\prime}}\right]_{+} .
\end{aligned}
$$

Here, $\varepsilon_{A}$ is the kinetic energy of a particle in the $A$-state, 
$\varphi_{A B}^{(E)}=\int \Psi_{A}^{*}(\vec{r}) \varphi^{(E)}(\vec{r}) \Psi_{B}(\vec{r}) d^{3} \vec{r}=$

$=-e \vec{E} \int \Psi_{A}^{*}(\vec{r}) \vec{r} \Psi_{B}(\vec{r}) d^{3} \vec{r} ;$

$V_{A B A^{\prime} B^{\prime}}=\int d^{3} \bar{r} \int d^{3} \vec{r}^{\prime} \Psi_{A}^{*}(\vec{r}) \Psi_{A^{\prime}}^{*}\left(\vec{r}^{\prime}\right) \frac{e^{2}}{\varepsilon_{L}\left|\vec{r}-\vec{r}^{\prime}\right|} \Psi_{B^{\prime}}^{*}\left(\vec{r}^{\prime}\right) \Psi_{A^{\prime}}^{*}(\vec{r}) ;$

$\Psi_{A}(\vec{r})$ is the wave-function of a free particle, $\varepsilon_{L}-$ dielectric constant of the considered crystal, $[\hat{a}, \hat{b}]_{+}=(1 / 2)(\hat{a} \hat{b}+\hat{b} \hat{a}) ; \Phi_{A B}^{(S)}-$ matrix element of the scattering micropotential. For example, for impurities with the charge $e_{I}$

$\Phi_{A B}^{(I)}=\sum_{j} \int d^{3} \vec{r} \Psi_{A}^{*}(\vec{r}) \frac{e_{I}}{\varepsilon_{L}\left|\vec{r}-\vec{r}_{j}\right|} \Psi_{B}(\vec{r})$

Introducing Hamiltonian (3) into the equation (2) and using standard commutation rules for fermions operators of generation and annihilation, one obtains this equation for the fluctuative part of the density matrix (see [1-3]):

$i \hbar \frac{\partial \rho_{A B}(t)}{\partial t}=\hbar \omega_{A B} \rho_{A B}(t)+e \sum_{\Gamma}\left\{\varphi_{A \Gamma}^{(E)} \rho_{\Gamma B}(t)-\rho_{A \Gamma}(t) \varphi_{\Gamma B}^{(E)}\right\}+$

$+e \sum_{\Gamma}\left\{\left[\varphi_{A \Gamma}(t), \rho_{\Gamma B}(t)\right]_{+}-\left[\rho_{A \Gamma}(t), \varphi_{\Gamma B}(t)\right]_{+}\right\} ;$

$\omega_{A B}=\omega_{A}-\omega_{B}$.

The matrix element of the screened scattering potential is

$$
\begin{aligned}
& \varphi_{A B}(t)=\Phi_{A B}^{(S)}(t)+(1 / e) \sum_{A^{\prime} B^{\prime}} V_{A B B^{\prime} A^{\prime}} \rho_{A^{\prime} B^{\prime}}(t) ; \\
& V_{A B B^{\prime} A^{\prime}}=\frac{e^{2}}{2 \pi^{2} \varepsilon_{L}} \int \frac{1}{q^{2}}\left(b_{\vec{q}}\right)_{A B}\left(b_{-\vec{q}}\right)_{B^{\prime} A^{\prime}} d^{3} \vec{q} ; \\
& \left(b_{\vec{q}}\right)_{A B}=\int \Psi_{A}^{*}(\vec{r}) \exp (i \vec{q} \vec{r}) \Psi_{B}(\vec{r}) d^{3} \vec{r} .
\end{aligned}
$$

Separate the density matrix into two parts: an averaged one and a fluctuation (here, angular brackets show the average with the help of non-equilibrium statistical operator (see, for instance, [4])

$$
\begin{aligned}
& \rho_{A B}(t)=\left\langle a_{B}^{+}(t) a_{A}(t)\right\rangle+\delta \rho_{A B}(t)= \\
& =f_{A B}(t)+\delta \rho_{A B}(t)=f_{A}(t) \delta_{A B}+\delta \rho_{A B}(t) .
\end{aligned}
$$

For spacially uniform system,

$$
f_{A B}(t)=f_{A}(t) \delta_{A B} \text {. }
$$

The average scattering potential is assumed to be zero:

$\varphi^{(S)}=\left\langle\varphi^{(S)}\right\rangle+\delta \varphi^{(S)}=\delta \varphi^{(S)}$.

Using Eq. (12) and the supposition $\left\langle\partial \rho_{A B}(t) / \partial t\right\rangle=\partial\left\langle\rho_{A B}(t)\right\rangle / \partial t$, perform the average of fundamental Eq. (7). One obtains:

$$
\begin{aligned}
& i \hbar \frac{\partial f_{A}(t)}{\partial t}=e \sum_{\Gamma}\left\{\varphi_{A \Gamma}^{(E)} f_{\Gamma A}(t)-f_{A \Gamma}(t) \varphi_{\Gamma A}^{(E)}\right\}+i \hbar \mathrm{St} f_{A} \\
& \text { St } f_{A}=-(2 e / \hbar) \operatorname{Im} \sum_{B}\left\langle\left[\delta \rho_{A B}(t), \delta \varphi_{B A}(t)\right]_{+}\right\rangle
\end{aligned}
$$

Composing the difference of Eqs. (7) and (14), we find the equation for the fluctuation of the density matrix:

$$
\begin{aligned}
& i \hbar \frac{\partial \delta \rho_{A B}}{\partial t}=\left(\varepsilon_{A}-\varepsilon_{B}\right) \delta \rho_{A B}+ \\
& +e \sum_{\Gamma}\left(\varphi_{A \Gamma}^{(E)} \delta \rho_{\Gamma B}-\delta \rho_{A \Gamma} \varphi_{\Gamma B}^{(E)}\right)+ \\
& +e \sum_{\Gamma}\left(\varphi_{A \Gamma} f_{\Gamma B}-f_{A \Gamma} \varphi_{\Gamma B}\right)+G_{A B},
\end{aligned}
$$

where

$G_{A B}=e \sum_{\Gamma}\left(\left[\varphi_{A \Gamma}, \delta \rho_{\Gamma B}\right]_{+}-\left\langle\left[\varphi_{A \Gamma}, \delta \rho_{\Gamma B}\right]_{+}\right\rangle-\right.$

$\left.-\left[\delta \rho_{A \Gamma}, \varphi_{\Gamma B}\right]_{+}+\left\langle\left[\delta \rho_{A \Gamma}, \varphi_{\Gamma B}\right]_{+}\right\rangle\right)$,

$\varphi^{(E)}(\vec{r})=-\vec{E} \cdot \vec{r} ; \quad \varphi_{A B}^{(E)}=-\vec{E} \cdot \vec{r}_{A B}$.

For $\vec{E}=\left(0,0, E_{z}\right)$ we have $\varphi_{A B}^{(E)}=E_{z} z_{A B}$.

Usually, one neglects in Eq. (15) the bilinear over fluctuations term $G_{A B}$ (that is so-called collisionless approximation for fluctuations). The field term in Eq. (15), which has the form

$e \sum_{\Gamma}\left(\varphi_{A \Gamma}^{(E)} \delta \rho_{\Gamma B}-\delta \rho_{A \Gamma} \varphi_{\Gamma B}^{(E)}\right)$,

is also usually omitted (see, for example, [2]). S, one declares that the electric field $E$ is small and neglect the conformable field term as negligible. But there appears a need to show some corresponding criterion which permits to omit this term. Usually, this criterion is not represented. Here, we don't neglect the discussed term.

If one uses the obvious representation of plane waves, he obtains:

$A \rightarrow \vec{k}_{A}=\left(k_{A x}, k_{A y}, k_{A z}\right)=\left(\vec{k}_{A \perp}, k_{A z}\right)$ etc.;

$\Psi_{A}(\vec{r}) \rightarrow \Psi_{\vec{k} A}(\vec{r})=L^{-3 / 2} \exp \left(i \vec{k}_{A} \vec{r}\right)=$

$=L^{-1} \exp \left(i \vec{k}_{A \perp} \vec{r}_{\perp}\right) \Psi_{k z A}(z)$.

Here, $\Psi_{k z A}(z)=L^{-1 / 2} \exp \left(i k_{A z} z\right)$. It follows from here:

$\varphi_{A \Gamma}^{(E)} \propto \delta_{A \Gamma}$,

and we come to the formal record:

$i \hbar \frac{\partial \delta \rho_{A B}(t)}{\partial t}=\left(\varepsilon_{A}-\varepsilon_{B}\right) \delta \rho_{A B}(t)+$

$+e\left(f_{B}-f_{A}\right) \varphi_{A B}(t)+$

$+e\left(\varphi_{A A}^{(E)}-\varphi_{B B}^{(E)}\right) \delta \rho_{A B}(t)+G_{A B}(t)$.

We can consider the term $G_{A B}(t)$ as a source of some part of a total "friction" force. 
Introduce the designation

$\varphi_{A A}^{(E)}-\varphi_{B B}^{(E)}=-E_{z}\left(z_{\vec{k} A, \vec{k} A}-z_{\vec{k} B, \vec{k} B}\right)$.

Represent Eq. (19) using the form

$i \hbar \frac{\partial \delta \rho_{A B}(t)}{\partial t}=\left\{\varepsilon_{A}-\varepsilon_{B}-e E_{z}[R(A, B)]\right\} \times$

$\times \delta \rho_{A B}(t)+e\left(f_{B}-f_{A}\right) \varphi_{A B}(t)$,

or

$i \hbar \frac{\partial \delta \rho_{A B}(t)}{\partial t}=\left(\bar{\varepsilon}_{A}-\bar{\varepsilon}_{B}\right) \delta \rho_{A B}(t)+e\left(f_{B}-f_{A}\right) \varphi_{A B}(t)$.

Let $k_{z A} \rightarrow k_{z}$ and $k_{z B} \rightarrow k_{z}+q_{z}$. Then the general structure of the form $R(A, B)$ (see Eq. (20)) and dimensionship of the considered term prompt the form close to

$$
E_{z}\left(z_{\vec{k} A, \vec{k} A}-z_{\vec{k} B, \vec{k} B}\right) \propto E_{z} q_{z} / k_{z}^{2} .
$$

The omitted here factor of proportionality requires a separate special calculation. That is found as nontrivial. If external electric field $\vec{E}$ (let that is homogeneous and constant in space) is applied to the system of noninteracting charged particles, the use of presentation of plane waves is not totally convenient and can be a source of some disturbance that appears in the limit case $\vec{E} \rightarrow 0$ (see [7]).

For the purpose to construct the appropriate form of the term $R(A, B)$, we proceed our consideration using some possible artificial way. At the fixed quantum number $k_{z A}$, choose the area $l_{A} \leq z \leq L_{A}$ and accept the model wave function $\Psi_{A}(\vec{r})$ as

$\Psi_{A}(\vec{r})=\Psi_{\vec{k} A}(\vec{r})=\frac{1}{L} \exp \left(i \vec{k}_{\perp A} \vec{r}_{\perp}\right) \Psi_{k z A}(z)$.

Here,

$\Psi_{k z A}(z)=\vartheta\left(z-l_{A}\right) \vartheta\left(L_{A}-z\right) C\left(k_{z A}\right) \cos \left(k_{z A} z\right)$;

$\vartheta(z)$ is the step function and

$C\left(k_{z A}\right)=\sqrt{\frac{2}{L_{A}-l_{A}}\left[1+\frac{\sin \left(2 k_{z A} L_{A}\right)-\sin \left(2 k_{z A} l_{A}\right)}{2 k_{z A}\left(L_{A}-l_{A}\right)}\right]^{-1}}$

The function (24) is normalized to unity:

$\int_{l A}^{L A} \Psi_{k z A}^{2}(z) d z=1$

Note that, inside the shown area, every function having the form (23), (24) is the self-function of the kinetic energy operator $-\hbar^{2} \nabla^{2} / 2 m$; the corresponding self-value for which is $\varepsilon_{A}=\hbar^{2}\left(k_{\perp A}^{2}+k_{z A}^{2}\right) / 2 m$. But for the whole set, all the proposed functions $\Psi_{k z}$ are not mutually orthogonal. We use these functions only to calculate the value $R(A, B)$.
Using the form (24), calculate the diagonal matrix element. Choose the lengths $L_{A}$ and $l_{A}$, as well as $L_{B}$ and $l_{B}$, satisfying the conditions

$\sin \left(2 k_{z A} L_{A}\right)=\sin \left(2 k_{z A} l_{A}\right)=$ $\sin \left(2 k_{z B} L_{B}\right)=\sin \left(2 k_{z B} l_{B}\right)=1$.

In addition, we accept the following relation: $L_{A}+l_{A}=L_{B}+l_{B}$. Then after several transformations and simplifications $\left(L_{A}>>l_{A}\right)$, we come to the following expression (see (22)):

$\bar{\omega}_{A B}=\omega_{A B}-e E_{z} R(A, B)=$

$=\frac{\hbar k_{A}^{2}}{2 m}-\frac{\hbar k_{B}^{2}}{2 m}-\frac{e E_{z}}{2 \hbar}\left[\frac{1}{k_{z A}}-\frac{1}{k_{z B}}\right]$.

The latter expression does not depend on the lengths $L$ and $l$. Naturally, the form (25) is not strictly correct, because we used the model wave-function. But we can consider it as acceptable at a sufficiently long length $L_{A}-l_{A}$.

Here, we have to notice that the form (24) of accepted wave-function is totally intuitive. Usage of this form is justified by final results for the calculated mobility that are totally transparent.

With the use of Eq. (7) and formulae (21)-(25), we execute a succession of calculations that repeat the standard order of steps (see, for instance, $[3,5]$ ) and come to the collision integral in the form:

$\mathrm{St}_{e-S} f_{A}=-\frac{e^{2}}{(2 \pi)^{3} \hbar} \times$

$\times \sum_{B} \int d^{3} \vec{q} \delta\left(\bar{\varepsilon}_{A}-\bar{\varepsilon}_{B}\right)\left|[\exp (i \vec{q} \vec{r})]_{A B}\right|^{2}\left\langle\varphi_{S}^{2}\right\rangle_{\vec{q}}\left(f_{A}-f_{B}\right)$.

Here, $\left\langle\varphi_{S}^{2}\right\rangle_{\vec{q}}$ is the correlator for Fouriercomponents of scattering potentials.

Let $A \rightarrow \vec{k}$ and $B \rightarrow \vec{k}+\vec{q}$; then in the representation of plane waves $[\exp (i \vec{q} \vec{r})]_{A B} \rightarrow \delta_{\vec{k}, \vec{k}+\vec{q}}$. In the course of the following calculations, we shall consider the case when scattering takes place with small transfer of momentum $(\langle q\rangle<\langle\langle k\rangle)$. Then it follows from Eq. (25):

$$
\begin{aligned}
& \bar{\varepsilon}_{A}-\bar{\varepsilon}_{B}=\varepsilon_{A}-\varepsilon_{B}-e E_{z}[R(A)-R(B)]= \\
& =\frac{\hbar^{2} k^{2}}{2 m}-\frac{\hbar(\vec{k}-\vec{q})^{2}}{2 m}+\frac{e E_{z} q_{z}}{2 k_{z}^{2}} .
\end{aligned}
$$

\section{Calculation of mobility}

For $\vec{k}$-representation, apply to both sides of kinetic equation (14) the operator $2(2 \pi)^{-3} \int \vec{k} d^{3} \vec{k}$. Let $u_{z}$ is $z$ component of the macroscopic drift velocity $\vec{u}$. 
Introducing mobility $\mu$ by the determination $u_{z}=\mu \cdot E_{z}$, we obtain the equation of the forces balance:

$$
e E_{z}=\frac{e u_{z}}{\mu}=\frac{2 \hbar}{(2 \pi)^{3} n} \int k_{z} \operatorname{St} f_{\vec{k}} d^{3} \vec{k} .
$$

Using the forms (26), (28) and the designation $\left\langle\varphi_{S}^{2}\right\rangle_{\vec{q}}=\Phi_{(S)} / q^{2 r+1}$, one finds after uncomplicated transformations:

$$
\frac{1}{\mu}=\frac{2 e \Phi_{(S)}}{(2 \pi)^{6} n u_{z}} \int d^{3} \vec{k} \int q_{z} d^{3} \vec{q} \delta\left(\bar{\varepsilon}_{\vec{k}}-\bar{\varepsilon}_{\vec{k}-\vec{q}}\right) f_{\vec{k}} / q^{2 r+1} .
$$

Here, $n$ is the density of band particles, forms for the functions $\Phi_{(S)}$ and numerical values of the parameter $r$ are determined by the choice of the mechanism of momentum relaxation.

Further, we restrict our consideration by the case of scattering of band carriers by uniformly distributed charged impurities with the density $n_{C I}$. For this case (see refs. [5, 6])

$$
r=3 / 2 ; \quad \Phi_{(S)}=\Phi_{(C I)}=32 \pi^{3} e^{2} n_{C I} / \varepsilon_{L}^{2} .
$$

Moreover, we will use the simplicative procedure for construction of the screened scattering potential. It introduces in (29) under the sign of integral the stepfunction $\vartheta\left(q-q_{0}\right)$, where $1 / q_{0}$ is the length of screening.

Implement the new designations:

$\vec{\kappa}_{\perp}=\vec{k}_{\perp} ; \kappa_{z}=k_{z}+e E_{z} m / 2 \hbar^{2} k_{z}^{2}$.

Then, the expression (27) takes the form

$\bar{\varepsilon}_{\vec{k}}-\bar{\varepsilon}_{\vec{k}-\vec{q}}=\hbar^{2} \vec{\kappa} \cdot \vec{q} / m-\hbar^{2} q^{2} / 2 m$.

At further calculation, one uses such simplification:

$\kappa_{z}=k_{z}+e E_{z} m / 4 \hbar^{2} k_{z}^{2} \rightarrow \kappa_{z}=$

$=k_{z}+e E_{z} m / 2 \hbar^{2}\left\langle k_{z}^{2}\right\rangle=k_{z}+3 e E_{z} / 4\langle\varepsilon\rangle$.

Here, the average energy is

$\langle\varepsilon\rangle=\frac{1}{4 \pi^{3} n} \int \varepsilon_{\vec{k}} f^{0}\left(\varepsilon_{\vec{k}}\right) d^{3} \vec{k}=\frac{3 k_{B} T}{2} \frac{F_{3 / 2}(\eta)}{F_{1 / 2}(\eta)}$,

$\eta=\varepsilon_{F} / k_{B} T$ is the dimensionless Fermi-energy,

$F_{r}(\eta)=\frac{1}{\Gamma(r+1)} \int_{0}^{\infty} \frac{w^{r} d w}{1+\exp (w-\eta)}$.

Carrying out in Eq. (29) the integration over components of vector $\vec{q}$, we obtain:

$$
\frac{1}{\mu}=\frac{e^{3} m n_{C I}}{\pi^{3} \hbar^{2} \varepsilon_{L}^{2} n u_{z}} \ln \left(\frac{q_{M}}{q_{0}}\right) \int \frac{\kappa_{z}}{\kappa^{3}(\vec{k})} f_{\vec{k}} d^{3} \vec{k},
$$

Where $q_{M} \propto 2 \sqrt{2 m\langle\varepsilon\rangle} / \hbar$. Considering the case $n=n_{C l}$, we come to the following record:

$$
E_{z}=E_{2} \int d^{3} \vec{K} f(\vec{K}) \frac{K_{z}+E_{z} / E_{1}}{\left[\left(K_{z}+E_{z} / E_{1}\right)^{2}+K_{\perp}^{2}\right]^{3 / 2}} .
$$

Further, the following designations are accepted:

$$
\begin{aligned}
& \vec{K}=\hbar \vec{k} / \sqrt{2 m k_{B} T} ; E_{2}=\frac{e^{3} \sqrt{2 m^{3} k_{B} T} \ln \left(q_{M} / q_{0}\right)}{\pi^{3} \varepsilon_{L}^{2} \hbar^{3}} ; \\
& E_{1}=E_{0} \frac{F_{3 / 2}(\eta)}{F_{1 / 2}(\eta)} ; E_{0}=\frac{2 \sqrt{2 m\left(k_{B} T\right)^{3}}}{e \hbar} .
\end{aligned}
$$

Introduce into the formula (35) the model form of the non-equilibrium function of distribution as the Fermi-function with the "shifted" argument:

$f_{\vec{k}}=f^{0}(\varepsilon(\vec{v}-\vec{u}))=f^{0}(\varepsilon(\hbar \vec{k} / m-\vec{u}))$.

Then,

$$
\begin{aligned}
& f_{\vec{k}}=\frac{1}{1+\exp \left[\left(\hbar k_{z} / \sqrt{2 m k_{B} T}-\sqrt{m} u_{z} / \sqrt{2 k_{B} T}\right)^{2}+\hbar^{2} k_{\perp}^{2} / 2 m k_{B} T-\eta\right]}, \\
& f(K)=\frac{1}{1+\exp \left\{\left[K_{z}-J\right]^{2}+K_{\perp}^{2}-\eta\right\}} . \\
& \text { Here, } \quad J=\sqrt{m} u_{z} / \sqrt{2 k_{B} T}=e n u_{z} / j_{0}=j_{z} / j_{0} .
\end{aligned}
$$

Below, one uses the designations:

$$
W=E_{z} / E_{0} ; \lambda(T)=\pi \frac{E_{2}}{E_{0}}=\frac{e^{4} m \ln \left(q_{M} / q_{0}\right)}{2 \pi^{2} \varepsilon_{L}^{2} \hbar^{2} k_{B} T} \frac{n_{C I}}{n} .
$$

Then, the relation between the dimensionless electric field $W$ and dimensionless current density $J$ takes the form:

$$
\begin{aligned}
& W=\lambda(T) \int d^{3} \vec{K} \frac{K_{z}+W F_{1 / 2}(\eta) / F_{3 / 2}(\eta)}{\left\{\left[K_{z}+W F_{1 / 2}(\eta) / F_{3 / 2}(\eta)\right]^{2}+K_{\perp}^{2}\right\}^{3 / 2}} \times \\
& \times \frac{1}{1+\exp \left\{\left[K_{z}-J\right]^{2}+K_{\perp}^{2}-\eta\right\}},
\end{aligned}
$$

or

$$
\begin{aligned}
& J=\xi-\frac{F_{1 / 2}(\eta)}{F_{3 / 2}(\eta)} \lambda(T) \times \\
& \times \int_{-\infty}^{\infty} d p \int_{0}^{\infty} d g \frac{p\left(g+p^{2}\right)^{-3 / 2}}{1+\exp \left[(p-\xi)^{2}+g-\eta\right]} .
\end{aligned}
$$

Here,

$\xi=\xi(W, J)=\frac{F_{1 / 2}(\eta)}{F_{3 / 2}(\eta)} W+J$.

Assuming the electric field to be small, perform linearization of the expression (40). One finds:

$J=\xi(W, J)\left[1-\frac{F_{1 / 2}(\eta)}{F_{3 / 2}(\eta)} S\right]$, 
where

$$
\begin{aligned}
& S(T)=2 \lambda(T) \int_{-\infty}^{\infty} d p \int_{0}^{\infty} d g \frac{p^{2}}{\left(g+p^{2}\right)^{3 / 2}} \times \\
& \times \frac{\exp \left[p^{2}+g-\eta(T)\right]}{\left\{1+\exp \left[p^{2}+g-\eta(T)\right]\right\}^{2}} .
\end{aligned}
$$

As a result, we come to the Ohm law in the following form $\left(W \rightarrow W_{1}\right)$ :

$$
W_{1}=J \frac{S}{1-S F_{1 / 2}(\eta) / F_{3 / 2}(\eta)} \text {. }
$$

Here, one obtains dimensionless specific conductivity (taking into account the evident dependence of the collisional integral on electric field) in the form:

$$
\bar{\sigma}_{1}=\frac{J}{W_{1}}=\frac{j_{z}}{E_{z}} \frac{E_{0}}{j_{0}}=\sigma_{1} \frac{E_{0}}{j_{0}}=\frac{1-S \cdot F_{1 / 2}(\eta) / F_{3 / 2}(\eta)}{S} .
$$

For the case of standard calculation, where the evident dependence of the collisional integral on electric field is not considered (so in the formula (35), one uses the change $K_{z}+E_{z} / E_{1} \rightarrow K_{z}$ ), the other record appears:

$$
\begin{aligned}
& W_{2}=\lambda(T) \int_{-\infty}^{\infty} d p \int_{0}^{\infty} d g \frac{p}{\left(g+p^{2}\right)^{3 / 2}} \times \\
& \times \frac{1}{1+\exp \left\{(p-J)^{2}+g-\eta\right\}} .
\end{aligned}
$$

After linearization of the expression (45), we obtain other form of Ohm law:

$$
\begin{aligned}
& W_{2}=2 \lambda(T) J \int_{-\infty}^{\infty} d p \int_{0}^{\infty} d g \frac{p^{2}}{\left(g+p^{2}\right)^{3 / 2}} \times \\
& \times \frac{\exp \left(p^{2}+g-\eta\right)}{\left[1+\exp \left(p^{2}+g-\eta\right)\right]^{2}},
\end{aligned}
$$

and come to the following expression for dimensionless specific conductivity:

$\bar{\sigma}_{2}=\frac{J}{W_{2}}=\frac{j_{z}}{E_{z}} \frac{E_{0}}{j_{0}}=\sigma_{2} \frac{E_{0}}{j_{0}}=\frac{1}{S}$.

One obtains from the formulae (44) and (47) the ratio of conductivities calculated by both mentioned ways:

$$
\begin{aligned}
& \sigma_{1}(T) / \sigma_{2}(T)= \\
= & \bar{\sigma}_{1}(T) / \bar{\sigma}_{2}(T)=1-S(T) \cdot F_{1 / 2}(\eta) / F_{3 / 2}(\eta) .
\end{aligned}
$$

\section{Conclusion}

Figure shows the results of calculations of the ratio of two specific conductivities. One of them (the value $\sigma_{1}$ ) relates to the consideration that takes into account the evident dependence of the collision integral on electric field. The other one (the value $\sigma_{2}$ ) relates to traditional approach that neglects the mentioned field dependence.

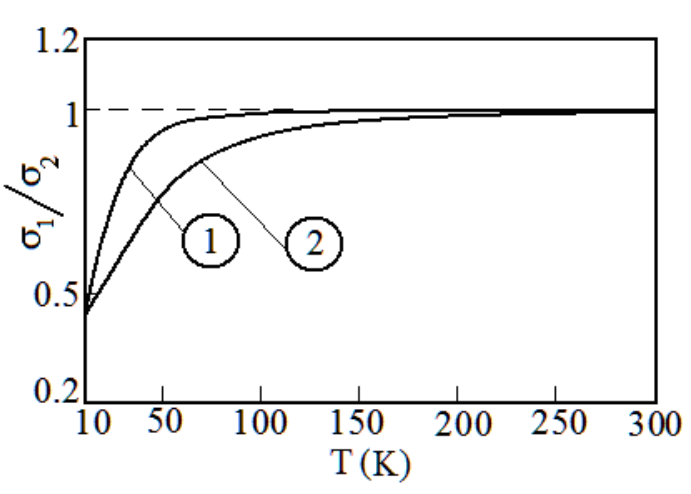

At construction of the shown figure, the following numerical values were used: $m=10^{-28} \mathrm{~g}, \varepsilon_{L}^{2}=150$, $\ln \left(q_{M} / q_{0}\right)=2.5$. The curve 1 relates to the case $n_{C I}=10^{16} \mathrm{~cm}^{-3}$, the curve $2-$ to the case $n_{C I}=10^{17} \mathrm{~cm}^{-3}$. It is seen for scattering with a small transfer of momentum that the introduced evident dependence of the collision integral results in decrease of the calculated conductivity, but doesn't disturb the Ohm law. The distinction of conductivities $\sigma_{1}$ and $\sigma_{2}$ is expressed clearer, if the temperature of carriers is lower and the density of scattering impurities is higher (practically, the less is the free path length). At a high temperature and low current density, the influence of the evident electric field, entered into collision integral, is not essential.

One can see the vast area of temperatures in presented figure, where neglecting the electrical term $e\left(\varphi_{A A}^{(E)}-\varphi_{B B}^{(E)}\right) \delta \rho_{A B}(t)$ in Eq. (21) and renouncement of the following complication of the collision integral is totally satisfactory. It follows from Eqs. (48) and (33) that possibility to neglect the evident dependence of the collision integral on the electric field $E$ is not bound with the amplitude of the field (as that is usually supposed, see $[1,2])$, but with mobility $\mu$ of carriers.

Introduce the designations:

$\mu_{1}=\sigma_{1} / e n ; \mu_{2}=\sigma_{2} / e n ; \mu_{0}=e \hbar / 2 m k_{B} T$.

Then, it follows from Eqs. (44) and (47):

$\mu_{(1)}=\mu_{(2)}\left[1-\frac{\mu_{0}}{\mu_{(2)}} \frac{3 k_{B} T}{2\langle\varepsilon\rangle}\right]$;

$\mu_{(1)} \approx \mu_{(2)} \rightarrow \mu$ at

$\mu>\mu_{0} \frac{3 k_{B} T}{2\langle\varepsilon\rangle}=\frac{3 e \hbar}{4 m\langle\varepsilon\rangle}$.

Represent the length of free motion $l_{(S c)}$ and deBroighle length :

$l_{(S c)}=\langle v\rangle \tau=\frac{\mu}{e} \sqrt{2 m\langle\varepsilon\rangle} ; l_{(d B)}=\frac{\hbar}{\sqrt{2 m\langle\varepsilon\rangle}} ;$

$\frac{l_{(d B)}}{l_{(S c)}}=\frac{e \hbar}{2 m\langle\varepsilon\rangle \mu}$. 

form:

Then, the condition (50) can be presented in the

$$
l_{(s c)} \gg l_{(d B)} .
$$

Therefore, the inequality (50) can be considered as the criterion of neglect by the evident dependence of the collision integral on the electric field. One can see that the usual concept of mobility is related to the small ratio $\left.\left.l_{(d B}\right) / l_{(s c}\right)$. Really, the concept of mobility, as it follows from (49) and (51), has a sense only at

$$
S(T) \cdot F_{1 / 2}(\eta) / F_{3 / 2}(\eta)<1
$$

From the physical viewpoint, the obtained results are evident. So, we can consider the model (24), as intuitive (like to every model) and as acceptable one.

\section{References}

1. Yu.L. Klimontovich, Statistical Physics. Nauka, Moscow, 1978 (in Russian).

2. E.M. Lifshits and L.P. Pitaevskiy, Physical Kinetics. Nauka, Moscow, 1979 (in Russian).

3. I.I. Boiko, Kinetics of Electron Gas Interacting with Fluctuating Potential. Naukova dumka, Kyiv, 1993 (in Russian).

4. D.N. Zubarev, Non-equilibrium Statistical Thermodynamics. Nauka, Moscow, 1971 (in Russian).

5. I.I. Boiko, Transport of Carriers in Semiconductors, V. Lashkaryov Institute of Semiconductor Physics, NAS of Ukraine, Kyiv, 2009 (in Russian).

6. A.I. Anselm, Introduction to the Theory of Semiconductors. Nauka, Moscow, 1978 (in Russian).

7. L.D. Landau and E.M. Lifshits, Quantum Mechanics. Nauka, Moscow, 1963 (in Russian). 